\title{
Power Allocation in Underlay Cognitive Radio Systems with Feasibility Detection
}

\author{
Sudhir Singh*, Paul D. Teal ${ }^{\dagger}$, Pawel A. Dmochowski ${ }^{\dagger}$ and Alan J. Coulson* \\ * Industrial Research Limited, Lower Hutt, New Zealand \\ $\dagger$ School of Engineering and Computer Science, Victoria University of Wellington, Wellington, New Zealand \\ Email:\{s.singh,a.coulson\}@irl.cri.nz, \{paul.teal,pawel.dmochowski\}@vuw.ac.nz
}

\begin{abstract}
We consider a cognitive radio system with $N$ secondary user (SU) pairs and a pair of primary users (PU). The SU power allocation problem is formulated as a capacity maximisation problem under PU and SU quality of service and SU peak power constraints. The problem is formulated as a geometric program, solved for both low- and high- signal-tointerference-and-noise ratio (SINR) regimes. We present a novel method of detecting and removing infeasible $S U$ quality of service constraints from the $\mathrm{SU}$ power allocation problem that results in considerably improved SU performance. Capacity cumulative distribution functions for Rayleigh fading channels are produced.
\end{abstract}

\section{INTRODUCTION}

A large number of papers have appeared on various aspects of cognitive radio (CR) systems, including fundamental information theoretic capacity limits (see, for example, [1-7]). In an underlay CR system the secondary users (SUs) protect the primary user (PU) by regulating their transmit power to maintain the PU receiver interference below a well defined threshold level. The limits on this received interference level at the PU receiver can be imposed by an average/peak constraint [2], or a minimum value for its signal-to-interference-and-noise ratio (SINR) [4]. Although it imposes an additional requirement that channel state information (CSI) be available, the advantage of using an SINR-based PU protection mechanism is that it removes the constant interference threshold, thus benefiting the SUs when the PU link is strong.

Power control in conventional wireless networks has been extensively studied in the literature [8-10]. Power control in CR systems presents its own unique challenges. In spectrum sharing applications, SU power must be allocated in a manner that achieves the goals of the CR system while not adversely affecting the operation of the PU. Generally the goals of the $\mathrm{CR}$ are not compatible with the goals of the PU; for instance, increasing SU power to increase SU capacity will tend to increase interference to the PU. There is a growing body of literature on power control and capacity of CR systems. In [11], soft sensing information was used for optimal power control to maximise capacity of one SU pair coexisting with one PU pair. The impacts of SU transmission power on the occurrence of spectrum opportunities and the reliability of opportunity detection was analysed in [12]. In [13], dynamic programming was used to develop a power control strategy for one SU pair under a Markov model of the PU's spectrum usage. Optimal power allocation strategies to achieve the ergodic capacity and the outage capacity of one SU pair coexisting with one PU pair under different types of power constraints and fading channel models were obtained in [6]. Power control using game-theoretic approaches have been proposed in $[14,15]$. Power control for CR systems using geometric programming have been proposed in [16-18]. A minimax approach was used in [18] to minimise the maximum transmit power for a CR system coexisting with a PU-Rx. The interference caused by a PU-Tx to the SU-Rxs was not considered in the problem formulation of [18]. In [16], a distributed approach was used for power allocation to maximise SU sum capacity under a peak interference constraint, but the approach did not include the interference caused by the PU-Tx in the analysis and the problem was only analysed for a high SINR scenario.

Convex optimisation methods are widely used in the design and analysis of communications systems. Many problems that arise in communications signal processing can be cast or converted into convex optimisation problems which allow analytical or numerical solutions to be calculated easily [19]. In [20], several problems for designing optimal dynamic resource allocation in CR systems are formulated and the key role that convex optimisation plays in finding the optimal solutions is demonstrated.

In [21], we formulated the SU power allocation problem as a capacity maximisation problem under PU and SU quality of service (QoS) and SU peak power constraints and showed that it can be solved using geometric programming and convex optimisation techniques. Unlike in [16-18], where the PU interference at each SU-Rx is neglected, the effect of the PU interference is evaluated in [21] by explicitly including it in the formulations. In this paper we extend the work of [21] and present a novel method of detecting and removing infeasible SU quality of service constraints from the SU power allocation problem that results in considerably improved SU performance. Solutions for both low and high SINR scenarios are presented. Capacity cumulative distribution functions (CDFs) for various channel conditions are obtained through solution of our optimisation problems.

\section{SYSTEM MODEL}

As shown in Fig. 1, we consider a cognitive radio system with a single PU and $N$ SU transmitters communicating simultaneously over a common channel to their respective receivers. Independent, point-to-point, flat Rayleigh fading channels are assumed for all links in the network. Let $g_{\mathrm{p}}=$ 


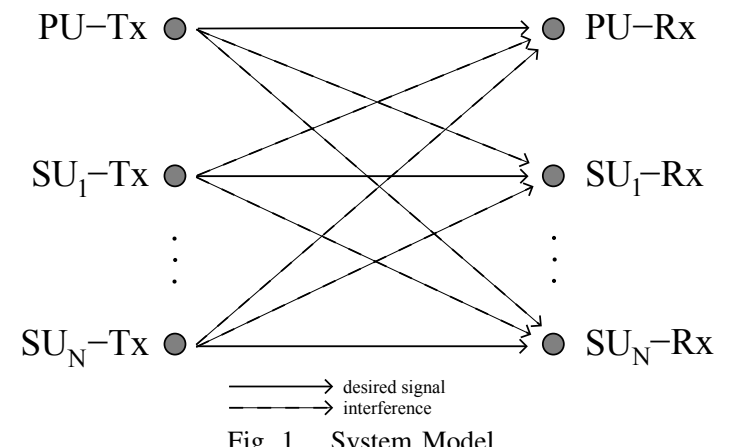

Fig. 1. System Model

$\left|h_{\mathrm{p}}\right|^{2}, g_{\mathrm{ss}}^{(i j)}=\left|h_{\mathrm{ss}}^{(i j)}\right|^{2}, g_{\mathrm{ps}}^{(j)}=\left|h_{\mathrm{ps}}^{(j)}\right|^{2}$ and $g_{\mathrm{sp}}^{(i)}=\left|h_{\mathrm{sp}}^{(i)}\right|^{2}$ denote the instantaneous channel powers of the PU-Tx to PU-Rx, SU-Tx $i$ to SU-Rx $j$, PU-Tx to SU-Rx $j$ and SUTx $i$ to PU-Rx links, respectively. For notational convenience we will denote $g_{\mathrm{s}}^{(i)}=g_{s s}^{(i i)}$. Furthermore, we assume that the channel powers for the PU and each of the $N$ SUs are independent and identically distributed (iid) and are governed by their corresponding parameters $\Omega_{\mathrm{p}}=\mathbb{E}\left(g_{p}\right), \Omega_{\mathrm{s}}=\mathbb{E}\left(g_{\mathrm{s}}\right)$, $\Omega_{\mathrm{ss}}=\mathbb{E}\left(g_{\mathrm{ss}}\right), \Omega_{\mathrm{ps}}=\mathbb{E}\left(g_{\mathrm{ps}}\right)$ and $\Omega_{\mathrm{sp}}=\mathbb{E}\left(g_{\mathrm{sp}}\right) . \mathbb{E}(\cdot)$ denotes the expectation operator. by

In our model the SINR at the $i$ th of $N$ SU receivers is given

$$
\gamma_{\mathrm{s}}^{(i)}=\frac{P_{\mathrm{s}}^{(i)} g_{\mathrm{s}}^{(i)}}{\sum_{j=1, j \neq i}^{N} P_{\mathrm{s}}^{(j)} g_{\mathrm{ss}}^{(i j)}+P_{\mathrm{p}} g_{\mathrm{ps}}^{(i)}+\sigma_{\mathrm{s}}^{2}}
$$

and that at the PU receiver by

$$
\gamma_{\mathrm{p}}=\frac{P_{\mathrm{p}} g_{\mathrm{p}}}{\sum_{i=1}^{N} P_{\mathrm{s}}^{(i)} g_{\mathrm{sp}}^{(i)}+\sigma_{\mathrm{p}}^{2}},
$$

where $P_{\mathrm{S}}^{(i)}$ and $P_{\mathrm{p}}$ are the $i$ th SU and PU transmit powers, respectively, and $\sigma_{\mathrm{s}}^{2}$ and $\sigma_{\mathrm{p}}^{2}$ are the additive white Gaussian noise (AWGN) variance at the $i$ th SU-Rx and PU-Rx, respectively. We also note that that there is a maximum transmit power constraint, $P_{\mathrm{s}, \max }^{(i)}$, on the SU transmitters which may be due either to regulatory or hardware limitations. This is denoted by

$$
P_{\mathrm{s}}^{(i)} \leq P_{\mathrm{s}, \max }^{(i)}
$$

Additionally, the vector $\mathbf{P}_{\mathrm{S}}$ is used to collectively refer to the set of SU transmit powers, i.e., $\mathbf{P}_{\mathrm{s}} \triangleq\left[P_{\mathrm{s}}^{(1)} \ldots P_{\mathrm{s}}^{(N)}\right]^{T}$.

In a cognitive radio system the secondary users are allowed to operate as long as they can guarantee a certain level of quality of service (QoS) to the primary user. Hence, in our analysis we impose an SINR constraint, $\gamma_{T}$, at the PU receiver

$$
\gamma_{\mathrm{p}} \geq \gamma_{\mathrm{T}}
$$

The SU sum capacity is given by

$$
C_{\Sigma}=\sum_{i=1}^{N} C_{i}
$$

where the individual capacity of the $i$ th $\mathrm{SU}$ is given by

$$
C_{i}=\log _{2}\left(1+\gamma_{\mathrm{s}}^{(i)}\right) \text {. }
$$

\section{SU POWER OPTIMISATION}

In this section, we aim to find the SU power allocation such that the SU capacity, $C_{\Sigma}$ is maximised while maintaining the PU receiver QoS above the threshold $\gamma_{\mathrm{T}}$, and keeping within the SU transmit power budget. We may optionally choose to set minimum SINR thresholds, $\gamma_{\mathrm{s}, \text { min }}^{(i)}$ on the $i$ th SU receiver. This represents a practical limitation on SU receivers below which the receivers fail to operate with acceptable performance. In our formulation we assume that all channel gains are known which allows us to obtain fundamental limits on capacity. However, in practise the channel gains would need to be estimated, hence the capacities obtained in this paper provide an upper bound. Mathematically we solve the following suite of optimisation problems.

1) SU Capacity Maximisation with SU QoS Constraints:

$$
\begin{array}{ll}
\underset{\mathbf{P}_{\mathrm{s}}}{\operatorname{maximise}} & C_{\Sigma} \\
\text { subject to } & \gamma_{\mathrm{p}} \geq \gamma_{\mathrm{T}} \\
& \gamma_{\mathrm{s}}^{(i)} \geq \gamma_{\mathrm{s}, \min }^{(i)}, \quad i=1, \ldots, N \\
& P_{\mathrm{s}}^{(i)} \leq P_{\mathrm{s}, \max }^{(i)}, \quad i=1, \ldots, N
\end{array}
$$

2) SU Capacity Maximisation without SU QoS Constraints:

$$
\begin{array}{ll}
\underset{\mathbf{P}_{\mathrm{s}}}{\operatorname{maximise}} & C_{\Sigma} \\
\text { subject to } & \gamma_{\mathrm{p}} \geq \gamma_{\mathrm{T}} \\
& P_{\mathrm{s}}^{(i)} \leq P_{\mathrm{s}, \max }^{(i)}, \quad i=1, \ldots, N
\end{array}
$$

From (3) and (4) it is obvious that maximising the objectives in (5) and (6) is equivalent to maximising

$$
\prod_{i=1}^{N}\left(1+\gamma_{\mathrm{s}}^{(i)}\right) \text {. }
$$

Problems (5) and (6) can be modified to minimisation problems by taking the reciprocal of the objectives. The suite of optimisation problems are nonlinear and non-convex and generally hard to solve [19]. We proceed by dividing our problem into high and low SINR scenarios.

\section{A. High SINR Scenario}

When the SINR is high, $C_{\Sigma}$ can be approximated by

$$
C_{\Sigma} \approx \log _{2}\left(\prod_{i=1}^{N} \gamma_{\mathrm{s}}^{(i)}\right)
$$

and so the optimisation problems (5) and (6) can be written in minimisation form as

$$
\begin{array}{cl}
\underset{\mathbf{P}_{\mathrm{s}}}{\operatorname{minimise}} & \prod_{i=1}^{N}\left(\frac{1}{\gamma_{\mathrm{s}}^{(i)}}\right) \\
\text { subject to } & \gamma_{\mathrm{p}} \geq \gamma_{\mathrm{T}} \\
& \gamma_{\mathrm{s}}^{(i)} \geq \gamma_{\mathrm{s}, \min }^{(i)}, \quad i=1, \ldots, N \\
& P_{\mathrm{s}}^{(i)} \leq P_{\mathrm{s}, \max }^{(i)}, \quad i=1, \ldots, N
\end{array}
$$

The second constraint in (9) is optional and only included if SU QoS constraints are required. 
Problem (9) falls into a class of optimisation problems known as geometric programs (GP). A GP is stated as the following optimisation problem.

$$
\begin{array}{ll}
\text { minimise } & f_{0}(\mathbf{x}) \\
\text { subject to } & f_{i}(\mathbf{x}) \leq 1, \quad i=1, \ldots, m \\
& h_{i}(\mathbf{x})=1, \quad i=1, \ldots, p,
\end{array}
$$

where $f_{0}, \ldots, f_{m}$ are in a form known as posynomials and $h_{1}, \ldots, h_{p}$ are referred to as monomials [19]. GPs are nonlinear, non-convex optimisation problems but can be transformed to convex optimisation problems by a logarithmic change of variables and by taking the logarithm of the objective and constraint functions [19]. The transformed problem can then be solved efficiently in polynomial time by interior point methods [22].

Through straightforward manipulation of the second and third constraints, problem (9) can be transformed into the standard form GP (10). Once in this form, they can be solved to obtain the optimum SU power allocation.

\section{B. Low SINR Scenario}

In the low SINR scenario our capacity maximisation optimisation problem is given by

$$
\begin{array}{cl}
\underset{\mathbf{P}_{\mathrm{s}}}{\operatorname{minimise}} & \prod_{i=1}^{N}\left(\frac{1}{1+\gamma_{\mathrm{s}}^{(i)}}\right) \\
\text { subject to } & \gamma_{\mathrm{p}} \geq \gamma_{\mathrm{T}} \\
& \gamma_{\mathrm{s}}^{(i)} \geq \gamma_{\mathrm{s}, \text { min }}^{(i)}, \quad i=1, \ldots, N \\
& P_{\mathrm{s}}^{(i)} \leq P_{\mathrm{s}, \text { max }}^{(i)}, \quad i=1, \ldots, N
\end{array}
$$

The second constraint in (11) is optional and only included if SU QoS constraints are required.

The objective in problem (11) is a ratio of posynomials and hence is not itself a posynomial. Optimisation problems of this nature are not GP and are known as Complementary GP $[23,24]$. Complementary GPs are non-convex problems but can be solved with an iterative technique known as the single condensation method $[23,24]$. In each iteration, the feasible point computed in the previous iteration is used to approximate the denominator of the objective monomial. Since a ratio of posynomial and monomial is a posynomial [19], the resulting problem is a GP. The procedure is repeated until the solution converges to an optimum of the original Complementary GP. It should be noted that convergence to a local or global minimum is possible. The posynomial is approximated with a monomial using the geometric-arithmetic mean inequality

$$
\sum_{i} \delta_{i} v_{i} \geq \prod_{i} v_{i}^{\delta_{i}}
$$

where $v_{i} \geq 0, \delta_{i} \geq 0$ and $\sum_{i} \delta_{i}=1$. If we let $u_{i}=\delta_{i} v_{i}$, then (12) can be written as

$$
\sum_{i} u_{i} \geq \prod_{i}\left(\frac{u_{i}}{\delta_{i}}\right)^{\delta_{i}} .
$$

Note that equality in (13) holds when $\delta_{i}=u_{i} / \sum_{i} u_{i}$. The term on the left hand side of (13) resembles the denominator of our objective, i.e. a sum of monomials. Hence, if we let $u_{i}\left(\mathbf{P}_{\mathrm{S}}\right)$ be the monomial terms of the denominator and $\delta_{i}=u_{i}\left(\mathbf{P}_{\mathrm{s}}\right) / \sum_{i} u_{i}\left(\mathbf{P}_{\mathrm{s}}\right)$, then from (13) it is clear that the denominator can be approximated around a feasible $\mathbf{P}_{\mathrm{S}}$ with a product of monomials. Since the approximation is always an under-estimator of the original posynomial, minimising the condensed objective guarantees that the solution moves towards a minimum of the original objective function.

For completeness, we present an algorithm that can be used for solving the low SINR capacity maximisation problem [10, 23, 24]:

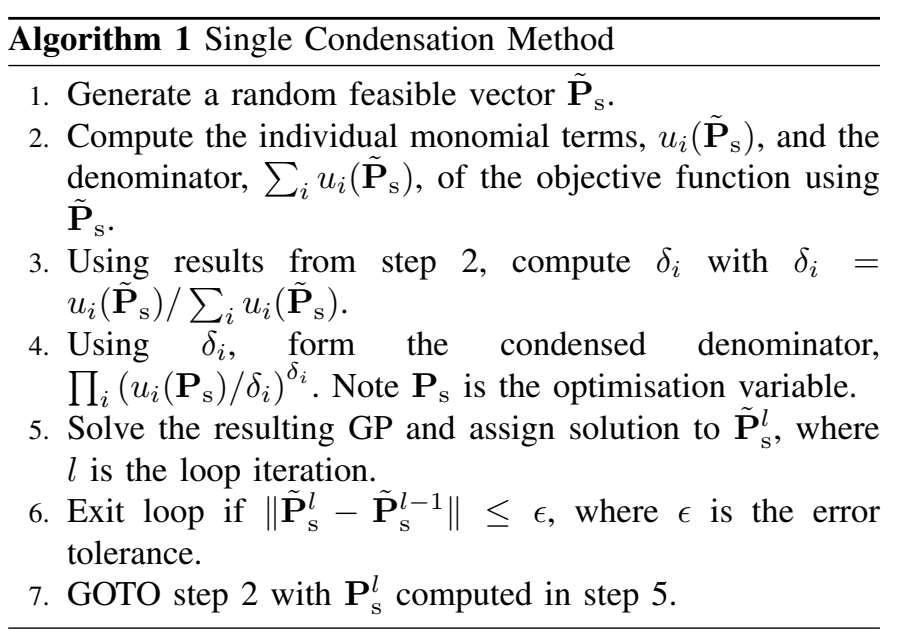

\section{SU POWER OPTIMISATION With FEASIBILITy DETECTION}

Optimisation problem (5) fails if at least one of the constraints is infeasible. This has an adverse effect on capacity since no SUs are able to access the channel if the QoS constraint cannot be met for any one (or more) SU. This, one SU which violates the QoS constraint has the potential of bringing down the whole system. It is clear that capacity can be improved by removing the violating SUs from the optimisation problem. In this section we formulate a method of detecting and removing the violating SUs from the optimisation problem. Our method is based on a feasibility detection technique, known as sum of infeasibilities, that is commonly used in interior point methods to find a strictly feasible starting point [19]. We form the problem

$$
\begin{array}{cl}
\underset{\mathbf{P}_{\mathrm{s}}, \mathbf{s}}{\operatorname{minimise}} & \mathbf{1}^{\mathrm{T}} \mathbf{s} \\
\text { subject to } & \frac{\gamma_{\mathrm{T}}}{\gamma_{\mathrm{p}}} \leq \mathbf{s}_{1} \\
& \frac{\gamma_{\mathrm{s}, \text { min }}^{(i)}}{\gamma_{\mathrm{s}}^{(i)}} \leq \mathbf{s}_{i+1}, \quad i=1, \ldots, N \\
& P_{\mathrm{s}}^{(i)} \leq P_{\mathrm{s}, \max }^{(i)}, \quad i=1, \ldots, N \\
& \mathbf{s} \succeq \mathbf{1},
\end{array}
$$

where 1 is a vector of length $N+1$ with all entries equal to one, $\mathbf{s} \in \mathbf{R}_{++}^{N+1}$ and $\succeq$ is the elementwise greater than or equal to comparison operator. For fixed $\mathbf{P}_{\mathrm{s}}$, the optimal values of $\mathbf{s}_{1}$ and $\mathbf{s}_{i+1}$ are $\max \left(\gamma_{\mathrm{T}} / \gamma_{\mathrm{p}}, 1\right)$ and $\max \left(\gamma_{\mathrm{s}, \min }^{(i)} / \gamma_{\mathrm{s}}^{(i)}, 1\right)$ 
respectively, so in problem (14), we are minimising the sum of the infeasibilities. The optimum value of (14) is $N+1$ and achieved if and only if the original set of constraints in (5) is feasible. It follows that all feasible constraints will have the corresponding element in the vector $\mathbf{s}$ equal to one. It should be noted that if $\mathbf{s}_{1}$ is not equal to one, i.e., the PU QoS constraint is infeasible, then problem (5) is infeasible and no SUs are admitted into the channel.

Let $\mathcal{I}$ represent the set of feasible SU QoS constraints determined from the solution of problem (14). We then solve the following SU power optimisation problem in which the violating SUs are removed

$$
\begin{array}{ll}
\underset{\mathbf{P}_{\mathrm{s}}}{\operatorname{maximise}} & C_{\Sigma} \\
\text { subject to } & \gamma_{\mathrm{p}} \geq \gamma_{\mathrm{T}} \\
& \gamma_{\mathrm{s}}^{(i)} \geq \gamma_{\mathrm{s}, \text { min }}^{(i)}, \quad i \in \mathcal{I} \\
& P_{\mathrm{s}}^{(i)} \leq P_{\mathrm{s}, \text { max }}^{(i)}, \quad i \in \mathcal{I}
\end{array}
$$

Problem (15) can be solved using methods presented in Section IV for the low and high SINR scenarios.

\section{Simulation Results and Discussion}

We now present simulation results of the optimisation problems formulated in Sections III and IV, specifically evaluating the CDFs of the resulting capacities. We consider a system with $N=3$ SUs. In all simulations we have set $P_{\mathrm{p}} / \sigma_{\mathrm{p}}^{2}=0 \mathrm{~dB}$ and $\Omega_{\mathrm{p}} / \sigma_{\mathrm{p}}^{2}=\Omega_{\mathrm{s}} / \sigma_{\mathrm{s}}^{2}=6.5 \mathrm{~dB}$, where we assume $\sigma_{\mathrm{p}}^{2}=\sigma_{\mathrm{s}}^{2}$. Simulations for problems (5) and (15) have $\gamma_{\mathrm{s}, \text { min }}^{(i)}=-10$ $\mathrm{dB}, i=1, \ldots, N$. For our simulations we parameterise the wireless channel as follows. The ratio of interference channel power to desired channel power is denoted by $c_{1}=\Omega_{\mathrm{sp}} / \Omega_{\mathrm{s}}$ and $c_{2}=\Omega_{\mathrm{ss}} / \Omega_{\mathrm{s}}$ parametrises the relative channel power of desired to interfering SU links. We consider the following three channel scenarios

1) Scenario A: Low Interference

In this scenario $c_{1}=c_{2}=0.1$ which corresponds to each receiver being approximately 3 times (assuming $1 / d^{2}$ path loss) further away from the interfering transmitters than its own transmitter. This results in low interference between all users, thus making the PU QoS constraint easy to satisfy.

2) Scenario $B$ : High Interference

In this scenario $c_{1}=c_{2}=0.9$ which corresponds to each receiver being approximately the same distance from all transmitters. This results in high interference among all users, thus making the PU QoS constraint difficult to satisfy.

3) Scenario $C$ : Low PU and High SU Interference In this scenario $c_{1}=0.1$ and $c_{2}=0.9$. Here the PU experiences low interference from the SUs since it is approximately 3 times further away from SU-Txs than the PU-Tx. As a result, the PU QoS constraint is easily satisfied. However, SU to SU interference is very prominent.

Results of our proposed methods are compared against the equal power allocation method and a power profile method analogous to the "poor man's waterfilling" method [25] where we allocate power proportionally to $g_{\mathrm{s}}^{(i)} / g_{\mathrm{sp}}^{(i)}$. We refer to these methods as ad hoc allocation methods. Note that the ad hoc allocation methods do not impose a minimum SU QoS requirement, hence a fair comparison is only possible against problems (6) and (15). Figures 2-4 show the SU sum capacity CDF obtained from optimisation problems (5), (6) and (15) for the three channel conditions with $\gamma_{\mathrm{T}}=2 \mathrm{~dB}$.

Figure 2 shows the SU sum capacity CDF of Scenario $A$ along with results of ad hoc allocation methods. Due to PU and SU QoS requirements of problem (5), we observe that around $50 \%$ of the time no SUs are able to access the channel. When problem (5) is resolved using the feasibility detection method-problem (15)—a marked improvement in performance is observed. The SU blocking probability drops to around $30 \%$ and similar performance to problem (6) is achieved. Furthermore, we see that the ad hoc allocation methods are outperformed by the GP methods.

Figure 3 shows the SU sum capacity CDF of Scenario $B$ along with results of ad hoc allocation methods. Due to $\mathrm{PU}$ and SU QoS requirements of problem (5), around $80 \%$ of the time no SUs are able to access the channel. Again, when problem (5) is resolved using the feasibility detection method-problem (15)—a marked improvement in performance is observed. The SU blocking probability drops to around $50 \%$. Due to the PU QoS requirements of problem (6), no SUs are able to transmit $47 \%$ of the time. We observe again that the ad hoc allocation methods are outperformed by the GP methods.

Figure 4 shows the SU sum capacity CDF of Scenario $C$ along with results of ad hoc allocation methods. Due to PU and SU QoS requirements of problem (5), around 57\% of the time no SUs are able to access the channel. Once again, when problem (5) is resolved using the feasibility detection method-problem (15)_performance is greatly improved. The SU blocking probability drops to around $35 \%$. Due to the PU QoS requirements of problem (6), no SUs are able to transmit $30 \%$ of the time. We also observe that again the ad hoc allocation methods are outperformed by the GP methods.

\section{CONCLUSions}

In this paper, we have formulated the SU power allocation problem in a CR system as a geometric program and obtained capacity CDFs in various channel conditions. We have included the effect of PU transmission in our formulations and studied the problem in both high and low SINR scenarios. More importantly, we have presented a method of detecting and removing infeasible SU quality of service constraints from the SU power allocation problem and have shown that applying this method results in considerably improved SU performance.

\section{REFERENCES}

[1] S. A. Jafar and S. Srinivasa, "Capacity limits of cognitive radio with distributed and dynamic spectral activity," IEEE J. Sel. Areas Commun., vol. 25, pp. 529-537, April 2007. 


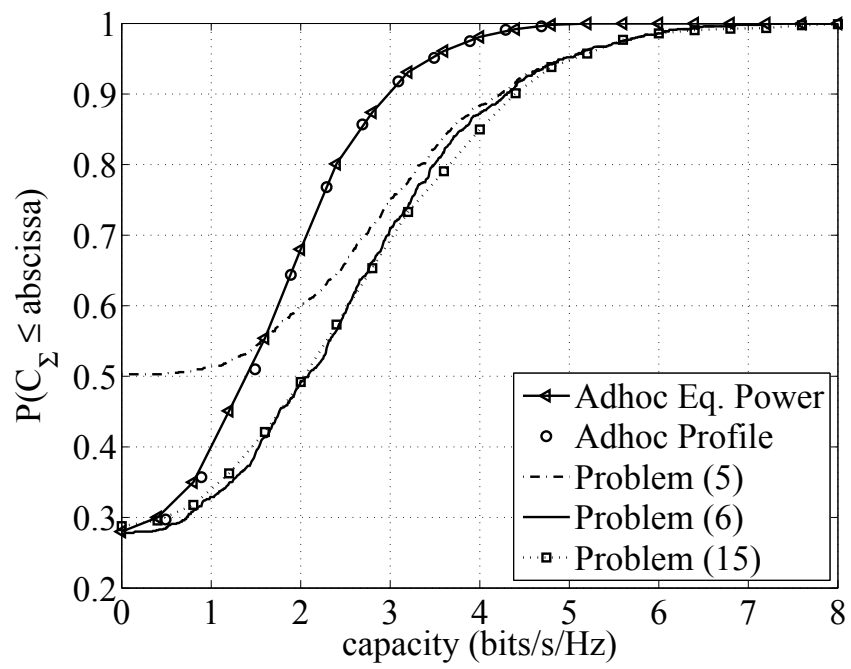

Fig. 2. $C_{\Sigma} \mathrm{CDF}$ for Scenario A, $\gamma_{\mathrm{T}}=2 \mathrm{~dB}$.

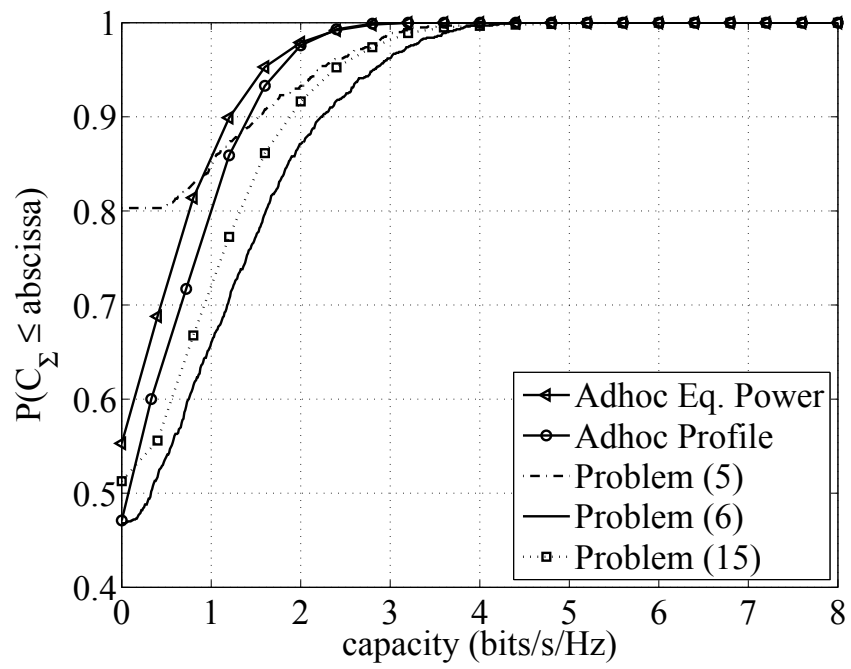

Fig. 3. $C_{\Sigma}$ CDF for Scenario $B, \gamma_{\mathrm{T}}=2 \mathrm{~dB}$.

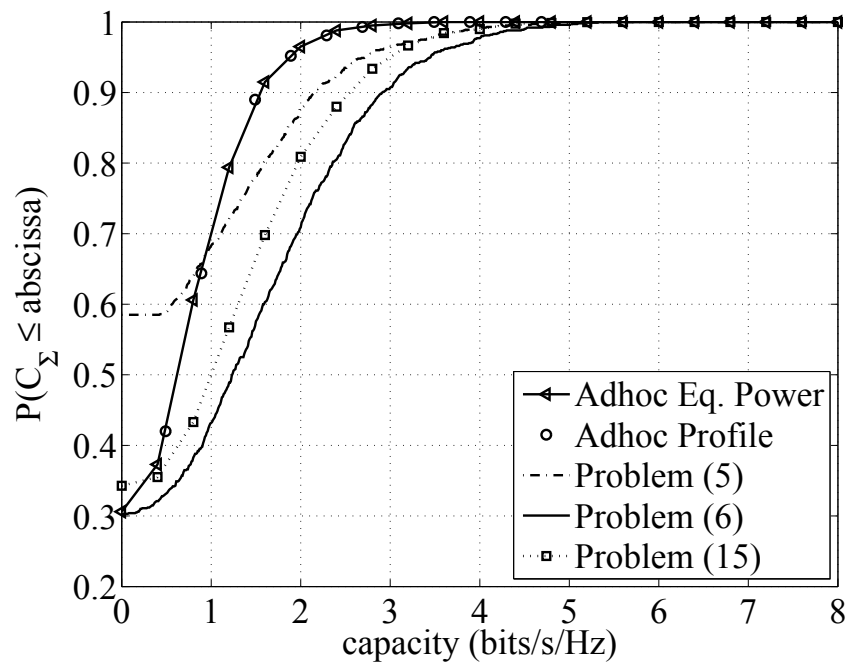

Fig. 4. $C_{\Sigma} \mathrm{CDF}$ for Scenario $C, \gamma_{\mathrm{T}}=2 \mathrm{~dB}$.
[2] A. Ghasemi and E. S. Sousa, "Fundamental limits of spectrum-sharing in fading environments," IEEE Trans. Wireless Commun., vol. 6, pp. 649-658, February 2007.

[3] L. Musavian and S. Aissa, "Fundamental capacity limits of spectrumsharing channels with imperfect feedback," in Proc. IEEE GLOBECOM 2007, November 2007, pp. 1385-1389.

[4] P. A. Dmochowski, H. A. Suraweera, P. J. Smith, and M. Shafi, "Impact of channel knowledge on cognitive radio system capacity," in Proc. IEEE VTC2010-Fall, September 2010, pp. 1-5.

[5] M. Shafi, H. A. Suraweera, P. J. Smith, and M. Faulkner, "Capacity limits and performance analysis of cognitive radio with imperfect channel knowledge," IEEE Trans. on Veh. Technol., January 2010.

[6] X. Kang, Y.-C. Liang, A. Nallanathan, H. K. Garg, and R. Zhang, "Optimal power allocation for fading channels in cognitive radio networks: Ergodic capacity and outage capacity," IEEE Trans. Wireless Commun., vol. 8, pp. 940-950, February 2009.

[7] C.-X. Wang, X. Hong, H.-H. Chen, and J. Thompson, "On capacity of cognitive radio networks with average interference power constraints," IEEE Trans. Wireless Commun., vol. 8, pp. 1620-1625, April 2009.

[8] C. Sung and W. Wong, "Power control and rate management for wireless multimedia cdma systems," IEEE Trans. Commun., vol. 49, no. 7, pp. $1215-1226$, July 2001.

[9] P. Gupta and P. R. Kumar, "The capacity of wireless networks," IEEE Trans. Inf. Theory, vol. 46, no. 2, pp. 388-404, March 2000.

[10] M. Chiang, C. W. Tan, D. Palomar, D. O'Neill, and D. Julian, "Power control by geometric programming," IEEE Trans. Wireless Commun., vol. 6, no. 7, pp. 2640-2651, July 2007.

[11] S. Srinivasa and S. A. Jafar, "Soft sensing and optimal power control for cognitive radio," IEEE Trans. on Wireless Commun., vol. 9, no. 12, pp. 3638-3649, December 2010.

[12] W. Ren, Q. Zhao, and A. Swami, "Power control in cognitive radio networks: How to cross a multi-lane highway," IEEE J. Sel. Areas Commun., vol. 27, no. 7, pp. 1283-1296, September 2009.

[13] Y. Chen, G. Yu, , Z. Zhang, H. Chen, and P. Qiu, "On cognitive radio networks with opportunistic power control strategies in fading channels," IEEE Trans. Wireless Commun., vol. 7, no. 7, pp. 2752-2761, July 2008.

[14] M. Alayesh and N. Ghani, "Game-theoretic approach for primarysecondary user power control under fast flat fading channels," IEEE Commun. Letters, vol. 15, no. 5, pp. 491-493, May 2011.

[15] P. Setoodeh and S. Haykin, "Robust transmit power control for cognitive radio," Proceedings of the IEEE, vol. 97, no. 5, pp. 915-939, May 2009.

[16] Q. Jin, D. Yuan, and Z. Guan, "Distributed geometric-programmingbased power control in cellular cognitive radio networks," in Proc. VTC 2009, April 2009, pp. 1-5.

[17] D. Li and X. Dai, "Power control in cooperative cognitive radio networks by geometric programming," in Proc. APCC 2009, October 2009, pp. $118-121$.

[18] L. Tang, H. Wang, and Q. Chen, "Power allocation with min-max fairness for cognitive radio networks," in Proc. WCNIS 2010, June 2010, pp. $478-482$.

[19] S. Boyd and L. Vandenberghe, Convex Optimization. Cambridge University Press, 2009.

[20] R. Zhang, Y.-C. Liang, and S. Cui, "Dynamic resource allocation in cognitive radio networks: A convex optimization perspective," arXiv, vol. abs/1001.3187, 2010.

[21] S. Singh, P. D. Teal, P. A. Dmochowski, and A. J. Coulson, "Interference management in cognitive radio systems - a convex optimisation approach," in Proc. ICC 2012, submitted.

[22] Y. Nesterov and A. Nemirovski, Interior Point Polynomial Methods in Convex Programming. SIAM Press, 1994.

[23] M. Avriel, Ed., Advances in Geometric Programming, ser. Mathematical Concepts and Methods in Science and Engineering. Plenum Press, 1980, vol. 21.

[24] C. S. Beightler and D. T. Philips, Applied Geometric Programming. Wiley, 1976.

[25] P. Smith, T. King, L. Garth, and M. Dohler, "A power scaling analysis of norm-based antenna selection techniques," IEEE Trans. on Wireless Commun., vol. 7, no. 8, pp. 3140-3149, August 2008. 